\title{
Determinant of Some Matrices of Field Elements
}

\author{
Yatsuka Nakamura ${ }^{1}$ \\ Shinshu University \\ Nagano, Japan
}

\begin{abstract}
Summary. Here, we present determinants of some square matrices of field elements. First, the determinat of $2 * 2$ matrix is shown. Secondly, the determinants of zero matrix and unit matrix are shown, which are equal to 0 in the field and 1 in the field respectively. Thirdly, the determinant of diagonal matrix is shown, which is a product of all diagonal elements of the matrix. At the end, we prove that the determinant of a matrix is the same as the determinant of its transpose.
\end{abstract}

MML identifier: MATRIX_7, version: 7.6.01 4.53.937

The articles [19], [26], [2], [27], [5], [4], [8], [24], [18], [17], [14], [6], [23], [7], [25], $[20],[21],[3],[12],[28],[10],[15],[16],[11],[13],[1],[9]$, and [22] provide the notation and terminology for this paper.

In this paper $n, i, l$ are natural numbers.

The following propositions are true:

(1) For every permutation $f$ of Seg 2 holds $f=\langle 1,2\rangle$ or $f=\langle 2,1\rangle$.

(2) For every finite sequence $f$ such that $f=\langle 1,2\rangle$ or $f=\langle 2,1\rangle$ holds $f$ is a permutation of Seg 2 .

(3) The permutations of 2-element set $=\{\langle 1,2\rangle,\langle 2,1\rangle\}$.

(4) For every permutation $p$ of $\operatorname{Seg} 2$ such that $p$ is a transposition holds $p=\langle 2,1\rangle$.

(5) Let $D$ be a non empty set, $f$ be a finite sequence of elements of $D$, and $k_{2}$ be a natural number. If $1 \leq k_{2}$ and $k_{2}<\operatorname{len} f$, then $f=\left(\operatorname{mid}\left(f, 1, k_{2}\right)\right)^{\frown}$ $\operatorname{mid}\left(f, k_{2}+1\right.$, len $\left.f\right)$.

\footnotetext{
${ }^{1}$ http://markun.cs.shinshu-u.ac.jp/kiso/staff/nakamura/index-e.html ynakamur@cs.shinshu-u.ac.jp 
(6) For every non empty set $D$ and for every finite sequence $f$ of elements of $D$ such that $2 \leq$ len $f$ holds $f=\left(f \uparrow\left(\operatorname{len} f-^{\prime} 2\right)\right)^{\frown} \operatorname{mid}\left(f\right.$, len $f-^{\prime} 1$, len $\left.f\right)$.

(7) For every non empty set $D$ and for every finite sequence $f$ of elements of $D$ such that $1 \leq \operatorname{len} f$ holds $f=\left(f\left\lceil\left(\operatorname{len} f-^{\prime} 1\right)\right)^{\wedge} \operatorname{mid}(f\right.$, len $f$, len $f)$.

(8) Let $a$ be an element of $A_{2}$. Given an element $q$ of the permutations of 2-element set such that $q=a$ and $q$ is a transposition. Then $a=\langle 2,1\rangle$.

(9) Let $n$ be a natural number, $a, b$ be elements of $A_{n}$, and $p_{2}, p_{1}$ be elements of the permutations of $n$-element set. If $a=p_{2}$ and $b=p_{1}$, then $a \cdot b=$ $p_{1} \cdot p_{2}$.

(10) Let $a, b$ be elements of $A_{2}$. Suppose that

(i) there exists an element $p$ of the permutations of 2-element set such that $p=a$ and $p$ is a transposition, and

(ii) there exists an element $q$ of the permutations of 2-element set such that $q=b$ and $q$ is a transposition.

Then $a \cdot b=\langle 1,2\rangle$.

(11) Let $l$ be a finite sequence of elements of $A_{2}$. Suppose that

(i) $\quad \operatorname{len} l \bmod 2=0$, and

(ii) for every $i$ such that $i \in \operatorname{dom} l$ there exists an element $q$ of the permutations of 2-element set such that $l(i)=q$ and $q$ is a transposition.

Then $\prod l=\langle 1,2\rangle$.

(12) For every field $K$ and for every matrix $M$ over $K$ of dimension 2 holds Det $M=M_{1,1} \cdot M_{2,2}-M_{1,2} \cdot M_{2,1}$.

Let $n$ be a natural number, let $K$ be a field, let $M$ be a matrix over $K$ of dimension $n$, and let $a$ be an element of $K$. Then $a \cdot M$ is a matrix over $K$ of dimension $n$.

The following three propositions are true:

(13) For every field $K$ and for all natural numbers $n, m$ holds $\operatorname{len}\left(\left(\begin{array}{ccc}0 & \ldots & 0 \\ \vdots & \ddots & \vdots \\ 0 & \ldots & 0\end{array}\right)_{K}^{n \times m}\right)=n$ and $\operatorname{dom}\left(\left(\begin{array}{ccc}0 & \ldots & 0 \\ \vdots & \ddots & \vdots \\ 0 & \ldots & 0\end{array}\right)_{K}^{n \times m}\right)=\operatorname{Seg} n$.

(14) Let $K$ be a field, $n$ be a natural number, $p$ be an element of the permutations of $n$-element set, and $i$ be a natural number. If $i \in \operatorname{Seg} n$, then $p(i) \in \operatorname{Seg} n$.

(15) For every field $K$ and for every natural number $n$ such that $n \geq 1$ holds $\operatorname{Det}\left(\left(\begin{array}{ccc}0 & \ldots & 0 \\ \vdots & \ddots & \vdots \\ 0 & \ldots & 0\end{array}\right)_{K}^{n \times n}\right)=0_{K}$

Let $x, y, a, b$ be sets. The functor $\operatorname{IFIN}(x, y, a, b)$ is defined by: 
(Def. 1) $\operatorname{IFIN}(x, y, a, b)=\left\{\begin{array}{l}a, \text { if } x \in y \\ b, \text { otherwise. }\end{array}\right.$

We now state the proposition

(16) For every field $K$ and for every natural number $n$ such that $n \geq 1$ holds $\operatorname{Det}\left(\left(\begin{array}{ccc}1 & & 0 \\ & \ddots & \\ 0 & & 1\end{array}\right)_{K}^{n \times n}\right)=1_{K}$.

Let $K$ be a field, let $n$ be a natural number, and let $M$ be a matrix over $K$ of dimension $n$. We say that $M$ being diagonal if and only if:

(Def. 2) For all natural numbers $i, j$ such that $i \in \operatorname{Seg} n$ and $j \in \operatorname{Seg} n$ and $i \neq j$ holds $M_{i, j}=0_{K}$.

One can prove the following propositions:

(17) Let $K$ be a field, $n$ be a natural number, and $A$ be a matrix over $K$ of dimension $n$. Suppose $n \geq 1$ and $A$ being diagonal. Then $\operatorname{Det} A=$ (the multiplication of $K) \circledast($ the diagonal of $A)$.

(18) Let $n$ be a natural number and $p$ be an element of the permutations of $n$-element set. Then $p^{-1}$ is an element of the permutations of $n$-element set.

Let us consider $n$ and let $p$ be an element of the permutations of $n$-element set. Then $p^{-1}$ is an element of the permutations of $n$-element set.

Next we state the proposition

(19) Let $n$ be a natural number, $K$ be a field, and $A$ be a matrix over $K$ of dimension $n$. Then $A^{\mathrm{T}}$ is a matrix over $K$ of dimension $n$.

Let $n$ be a natural number, let $K$ be a field, and let $A$ be a matrix over $K$ of dimension $n$. The functor $A^{\mathrm{T}}$ yields a matrix over $K$ of dimension $n$ and is defined as follows:

(Def. 3) $\quad A^{\mathrm{T}}=(A \text { qua matrix over } K)^{\mathrm{T}}$.

The following proposition is true

(20) For every group $G$ and for all finite sequences $f_{1}, f_{2}$ of elements of $G$ holds $\left(\prod\left(f_{1} \frown f_{2}\right)\right)^{-1}=\left(\prod f_{2}\right)^{-1} \cdot\left(\prod f_{1}\right)^{-1}$.

Let $G$ be a group and let $f$ be a finite sequence of elements of $G$. The functor $f^{-1}$ yields a finite sequence of elements of $G$ and is defined by:

(Def. 4) $\operatorname{len}\left(f^{-1}\right)=\operatorname{len} f$ and for every natural number $i$ such that $i \in \operatorname{Seg}$ len $f$ holds $\left(f^{-1}\right)_{i}=\left(f_{i}\right)^{-1}$.

One can prove the following propositions:

(21) For every group $G$ holds $\left(\varepsilon_{(\text {the carrier of } G)}\right)^{-1}=\varepsilon_{(\text {the carrier of } G)}$.

(22) For every group $G$ and for all finite sequences $f, g$ of elements of $G$ holds $\left(f^{\frown} g\right)^{-1}=\left(f^{-1}\right)^{\frown} g^{-1}$.

(23) For every group $G$ and for every element $a$ of $G$ holds $\langle a\rangle^{-1}=\left\langle a^{-1}\right\rangle$. 
(24) For every group $G$ and for every finite sequence $f$ of elements of $G$ holds $\prod\left(f^{\frown}(\operatorname{Rev}(f))^{-1}\right)=1_{G}$.

(25) For every group $G$ and for every finite sequence $f$ of elements of $G$ holds $\prod\left(\left((\operatorname{Rev}(f))^{-1}\right)^{\frown} f\right)=1_{G}$.

(26) For every group $G$ and for every finite sequence $f$ of elements of $G$ holds $\left(\prod f\right)^{-1}=\prod\left((\operatorname{Rev}(f))^{-1}\right)$.

(27) Let $I_{1}$ be an element of the permutations of $n$-element set and $I_{2}$ be an element of $A_{n}$. If $I_{2}=I_{1}$ and $n \geq 1$, then $I_{1}{ }^{-1}=I_{2}{ }^{-1}$.

(28) Let $n$ be a natural number and $I_{3}$ be an element of the permutations of $n$-element set. If $n \geq 1$, then $I_{3}$ is even iff $I_{3}{ }^{-1}$ is even.

(29) Let $n$ be a natural number, $K$ be a field, $p$ be an element of the permutations of $n$-element set, and $x$ be an element of $K$. If $n \geq 1$, then $(-1)^{\operatorname{sgn}(p)} x=(-1)^{\operatorname{sgn}\left(p^{-1}\right)} x$.

(30) Let $K$ be a field and $f_{1}, f_{2}$ be finite sequences of elements of $K$. Then (the multiplication of $K) \circledast\left(f_{1} \frown f_{2}\right)=\left((\right.$ the multiplication of $\left.K) \circledast\left(f_{1}\right)\right)$. ((the multiplication of $\left.K) \circledast\left(f_{2}\right)\right)$.

(31) Let $K$ be a field and $R_{1}, R_{2}$ be finite sequences of elements of $K$. Suppose $R_{1}$ and $R_{2}$ are fiberwise equipotent. Then (the multiplication of $K$ ) $\circledast$ $\left(R_{1}\right)=($ the multiplication of $K) \circledast\left(R_{2}\right)$.

(32) Let $n$ be a natural number, $K$ be a field, $p$ be an element of the permutations of $n$-element set, and $f, g$ be finite sequences of elements of $K$. If $n \geq 1$ and len $f=n$ and $g=f \cdot p$, then $f$ and $g$ are fiberwise equipotent.

(33) Let $n$ be a natural number, $K$ be a field, $p$ be an element of the permutations of $n$-element set, and $f, g$ be finite sequences of elements of $K$. Suppose $n \geq 1$ and len $f=n$ and $g=f \cdot p$. Then (the multiplication of $K) \circledast f=($ the multiplication of $K) \circledast g$.

(34) Let $n$ be a natural number, $K$ be a field, $p$ be an element of the permutations of $n$-element set, and $f$ be a finite sequence of elements of $K$. If $n \geq 1$ and len $f=n$, then $f \cdot p$ is a finite sequence of elements of $K$.

(35) Let $n$ be a natural number, $K$ be a field, $p$ be an element of the permutations of $n$-element set, and $A$ be a matrix over $K$ of dimension $n$. If $n \geq 1$, then $p^{-1}$-Path $A^{\mathrm{T}}=(p-\operatorname{Path} A) \cdot p^{-1}$.

(36) Let $n$ be a natural number, $K$ be a field, $p$ be an element of the permutations of $n$-element set, and $A$ be a matrix over $K$ of dimension $n$. Suppose $n \geq 1$. Then (the product on paths of $\left.A^{\mathrm{T}}\right)\left(p^{-1}\right)=$ (the product on paths of $A)(p)$.

(37) Let $n$ be a natural number, $K$ be a field, and $A$ be a matrix over $K$ of dimension $n$. If $n \geq 1$, then $\operatorname{Det} A=\operatorname{Det}\left(A^{\mathrm{T}}\right)$. 


\section{REFERENCES}

[1] Grzegorz Bancerek. Cardinal numbers. Formalized Mathematics, 1(2):377-382, 1990.

[2] Grzegorz Bancerek. The fundamental properties of natural numbers. Formalized Mathematics, 1(1):41-46, 1990.

[3] Grzegorz Bancerek and Krzysztof Hryniewiecki. Segments of natural numbers and finite sequences. Formalized Mathematics, 1(1):107-114, 1990.

[4] Czesław Byliński. Binary operations. Formalized Mathematics, 1(1):175-180, 1990.

[5] Czesław Byliński. Functions and their basic properties. Formalized Mathematics, 1(1):55$65,1990$.

[6] Czesław Byliński. Functions from a set to a set. Formalized Mathematics, 1(1):153-164, 1990.

[7] Czesław Byliński. Partial functions. Formalized Mathematics, 1(2):357-367, 1990.

[8] Czesław Byliński. Some basic properties of sets. Formalized Mathematics, 1(1):47-53, 1990.

[9] Czesław Byliński. Some properties of restrictions of finite sequences. Formalized Mathematics, 5(2):241-245, 1996.

[10] Agata Darmochwał. Finite sets. Formalized Mathematics, 1(1):165-167, 1990.

[11] Katarzyna Jankowska. Matrices. Abelian group of matrices. Formalized Mathematics, 2(4):475-480, 1991.

[12] Katarzyna Jankowska. Transpose matrices and groups of permutations. Formalized Mathematics, 2(5):711-717, 1991.

[13] Jarosław Kotowicz. Functions and finite sequences of real numbers. Formalized Mathematics, 3(2):275-278, 1992.

[14] Eugeniusz Kusak, Wojciech Leończuk, and Michał Muzalewski. Abelian groups, fields and vector spaces. Formalized Mathematics, 1(2):335-342, 1990.

[15] Yatsuka Nakamura and Roman Matuszewski. Reconstructions of special sequences. Formalized Mathematics, 6(2):255-263, 1997. http://fm.mizar.org/1997-6/pdf6-2/jordan3.pdf

[16] Takaya Nishiyama and Yasuho Mizuhara. Binary arithmetics. Formalized Mathematics, 4(1):83-86, 1993.

[17] Library Committee of the Association of Mizar Users. Binary operations on numbers. To appear in Formalized Mathematics.

[18] Andrzej Trybulec. Subsets of complex numbers. To appear in Formalized Mathematics.

[19] Andrzej Trybulec. Tarski Grothendieck set theory. Formalized Mathematics, 1(1):9-11, 1990.

[20] Andrzej Trybulec and Agata Darmochwał. Boolean domains. Formalized Mathematics, $1(\mathbf{1}): 187-190,1990$.

[21] Wojciech A. Trybulec. Binary operations on finite sequences. Formalized Mathematics, 1(5):979-981, 1990.

[22] Wojciech A. Trybulec. Groups. Formalized Mathematics, 1(5):821-827, 1990.

[23] Wojciech A. Trybulec. Pigeon hole principle. Formalized Mathematics, 1(3):575-579, 1990.

[24] Wojciech A. Trybulec. Vectors in real linear space. Formalized Mathematics, 1(2):291296,1990

[25] Wojciech A. Trybulec. Lattice of subgroups of a group. Frattini subgroup. Formalized Mathematics, 2(1):41-47, 1991.

[26] Zinaida Trybulec. Properties of subsets. Formalized Mathematics, 1(1):67-71, 1990.

[27] Edmund Woronowicz. Relations and their basic properties. Formalized Mathematics, 1(1):73-83, 1990.

[28] Katarzyna Zawadzka. The product and the determinant of matrices with entries in a field. Formalized Mathematics, 4(1):1-8, 1993. 